\title{
ELEMENTARY OSCILLATION CRITERIA FOR A THREE TERM RECURRENCE RELATION WITH OSCILLATORY COEFFICIENT SEQUENCE
}

\author{
GUANG ZHANG AND SUI SUN CHENG
}

\begin{abstract}
Alostract. Qualitative properties of recurrence relations with coefficients taking on both positive and. negative values are difficult to obtain since mathematical tools are scarce. In this note we start from scratch and obtain a number of oscillation criteria for one such relation: $x_{n+1}-x_{n}+$ $p_{n} x_{n-r} \leq 0$.
\end{abstract}

\section{Introduction}

Sequences $\left\{x_{n}\right\}_{n=-r}^{\infty}$ that satisfy recurrence relations of the form

$$
x_{n+1}-x_{n}+p_{n} x_{n-r} \leq 0, \quad n=0,1,2, \ldots,
$$

where $\tau$ is a nonnegative integer, have been studied by many authors and their oscillatory properties are known to some extent when the coefficient sequence $\left\{p_{n}\right\}_{n=0}^{\infty}$ is nonnegative (see e. g. [1]). When $\left\{p_{n}\right\}$ takes on both positive as well as negative values, however, to the best of our knowledge, there seem to be only a limited number of studies $[2,3]$ which are concerned with the corresponding oscillatory properties. In some senses, this is not surprising since mathematical tools are not available for dealing with such relations.

In this note, we will start from scratch and derive a number of elementary nonexistence criteria for eventually positive solutions of (1). To motivate the results that follow, let us first consider the case where $\tau=0$. Suppose the sequence $\left\{p_{n}\right\}$ has a subsequence $\left\{p_{n(i)}\right\}_{i=1}^{\infty}$ which satisfies $p_{n(i)} \geq 1$ for $n \geq 1$. If there is an eventually positive solution $\left\{x_{n}\right\}$ of (1), then there is a positive integer $I$ such that $x_{k}>0$ for $k \geq n(I)$. In view of (1), we see that

$$
0<x_{n(I)+1} \leq x_{n(I)}-p_{n(I)} x_{n(I)}=x_{n(I)}\left(1-p_{n(I)}\right),
$$

which is a contradiction. In other words, if $\left\{p_{n}\right\}$ has a subsequence $\left\{p_{n(i)}\right\}_{i=1}^{\infty}$ which satisfies $p_{n(i)} \geq 1$ for $i \geq 1$, such an eventually positive solution cannot exist. This conclusion is sharp in the sense that when (1) is replaced by

$$
x_{n+1}-x_{n}+p_{n} x_{n}=0, \quad n=0,1,2, \ldots,
$$

Received December 15, 1997.

1991 Mathematics Subject Classification. Primary 39A10.

Key words and phrases. Recurrence relation, eventually positive solution. 
then assuming $p_{n}<1$ for $n \geq 0$, the sequence $\left\{x_{n}\right\}$ defined by

$$
x_{0}=1, x_{n}=\Pi_{i=0}^{n-1}\left(1-p_{i}\right), n=1,2, \ldots,
$$

is an eventually positive solution of (2).

When $\tau>0$, we may argue along similar lines and come up with a number of nonexistence criteria which are also novel. A preparatory result is needed.

Lemma 1. Let $\left\{x_{n}\right\}_{n=-\tau}^{\infty}$ be a solution of (1). If $p_{k} x_{k-\tau} \geq 0$ for any nonnegative integer $k$, then $x_{k} \geq x_{k+1}$. If $p_{m-\tau}, p_{m-\tau+1}, \ldots, p_{m+j} \geq 0$, and $x_{m-2 \tau}, \ldots, x_{m-\tau-1} \geq 0$ for some $m \geq \tau$ and some $j \in\{0,1, \ldots, \tau\}$, then

$$
x_{m+j+1} \leq\left(1-p_{m}-\cdots-p_{m+j}\right) x_{m}
$$

Proof. First of all, in view of (1), we have $x_{k} \geq x_{k+1}+p_{k} x_{k-\tau} \geq x_{k+1}$. Thus if $p_{m-\tau}, p_{m-\tau+1}, \ldots, p_{m+j} \geq 0$, and $x_{m-2 \tau}, \ldots, x_{m-\tau-1} \geq 0$ for some $m \geq \tau$ and some $j \in\{0,1, \ldots, \tau\}$, then

$$
x_{m-\tau} \geq x_{m-\tau+1} \geq \cdots \geq x_{m} .
$$

By summing (1) from $m$ to $m+j$, we see further that

$$
\begin{aligned}
0 \geq & x_{m+j+1}-x_{m}+p_{m} x_{m-\tau}+p_{m+1} x_{m-\tau+1}+\cdots+p_{m+j} x_{m-\tau+j} \\
& \geq x_{m+j+1}+\left(-1+p_{m}+\cdots+p_{m+j}\right) x_{m}
\end{aligned}
$$

as desired.

Before we derive our first result, let us go through a simple situation first. Suppose $\left\{p_{n}\right\}$ has a subsequence $\left\{p_{n(i)}\right\}_{i=1}^{\infty}$ which satisfies

$$
p_{n(i)-\tau}, p_{n(i)-\tau+1}, \ldots, p_{n(i)-1} \geq 0
$$

and

$$
p_{n(i)} \geq 1
$$

for $i \geq 1$, then there cannot be any eventually positive solutions of (1).

Indeed, assume to the contrary that $\left\{x_{n}\right\}$ is an eventually positive solution of (1). Then there exists a positive integer $I$ such that $x_{k}>0$ for $k \geq n(I)-2 \tau$. By means of Lemma 1 , if we take $j=0$, then

$$
0<x_{n(I)+1} \leq x_{n(I)}\left(1-p_{n(I)}\right) \leq 0
$$

which is a contradiction.

We remark that if $\tau=0$, the conditions $p_{n(i)-1}, p_{n(i)-2}, \ldots, p_{n(i)-\tau} \geq 0$ are vacuously true. Similar remarks will hold for all later results.

In case the condition that $p_{n(i)} \geq 1$ for $i \geq 1$ is not satisfied, we may check if the conditions in the following more general result are satisfied. 
Theorem 1. Let $j \in\{0,1, \ldots, \tau\}$. If $\left\{p_{n}\right\}$ has a subsequence $\left\{p_{n(i)}\right\}_{i=1}^{\infty}$ which
isfies satisfies

$$
p_{n(i)-\tau}, p_{n(i)-\tau+1}, \ldots, p_{n(i)+j} \geq 0
$$

and

$$
p_{n(i)}+p_{n(i)+1}+\cdots+p_{n(i)+j} \geq 1
$$

for $i \geq 1$, then there cannot be any eventually positive solutions of (1).

Indeed, assume to the contrary that $\left\{x_{n}\right\}$ is an eventually positive solution of (1). Then there exists a positive integer $I$ such that $x_{k}>0$ for $k \geq n(I)-2 \tau$. By means of
Lemma 1,

which is a contradiction.

$$
0<x_{n(I)+j+1} \leq\left(1-p_{n(I)}-\cdots-p_{n(I)+j}\right) x_{n(I)} \leq 0,
$$

In particular, if we take $j=\tau$ in the above Theorem, we see that when $\left\{p_{n}\right\}$ has a subsequence $\left\{p_{n(i)}\right\}_{i=1}^{\infty}$ which satisfies

$$
p_{n(i)-\tau}, p_{n(i)-\tau+1}, \ldots, p_{n(i)+\tau} \geq 0 \text { and } p_{n(i)}+p_{n(i)+1}+\cdots+p_{n(i)+\tau} \geq 1
$$

for $i \geq 1$, then (1) cannot have any eventually positive solutions.

As an example, consider the equation

$$
x_{n+1}-x_{n}+q_{n} x_{n-1}=0, \quad n=0,1,2, \ldots,
$$

where $q_{n}=-1$ except that $q_{k^{2}-1}=q_{k^{2}}=1$ for $k=1,2, \ldots$. Then Theorem 1 implies that every solution of this equation oscillates. This can also be seen directly since

$$
\begin{aligned}
x_{k^{2}+1}= & x_{k}^{2}-q_{k^{2}} x_{k^{2}-1}=x_{k^{2}-1}-q_{k^{2}-1} x_{k^{2}-2}-q_{k^{2}} x_{k^{2}-1} \\
& =x_{k^{2}-1}-x_{k^{2}-2}-x_{k^{2}-1}=-x_{k^{2}-2}
\end{aligned}
$$

for all $k=1,2,3 \ldots$

To motivate our second result, let us assume that $\left\{p_{n}\right\}$ has a subsequence $\left\{p_{n(i)}\right\}_{i=1}^{\infty}$ which satisfies

$$
p_{n(i)-2 \tau}, p_{n(i)-2 \tau+1}, \ldots, p_{n(i)-\tau-1} \geq 0, \text { and } 0 \leq p_{n(i)-\tau}, p_{n(i)-\tau+1}, \ldots, p_{n(i)-1}<1
$$

for $i \geq 1$. If (1) has an eventually positive solution such that $x_{k}>0$ for $k \geq n(I)-3 \tau$ for some positive integer $I$. Then in view of Lemma 1 , we have

so that.

$$
x_{n(I)} \leq\left(1-p_{n(I)-1}\right) x_{n(I)-1}, \ldots, x_{n(I)-\tau+1} \leq\left(1-p_{n(I)-\tau}\right) x_{n(I)-\tau}
$$

In view of (1),

$$
x_{n(I)} \leq \Pi_{k=1}^{\tau}\left(1-p_{n(I)-k}\right) x_{n(I)-\tau} .
$$

$$
0<x_{n(I)+1} \leq x_{n(I)}-p_{n(I)} x_{n(I)-\tau} \leq\left[\Pi_{k=1}^{\tau}\left(1-p_{n(I)-k}\right)-p_{n(I)}\right] x_{n(I)-\tau} \leq 0
$$


which is a contradiction, provided that $p_{n(I)} \geq \Pi_{k=1}^{\tau}\left(1-p_{n(I)-k}\right)$.

Theorem 2. If $\left\{p_{n}\right\}$ has a subsequence $\left\{p_{n(i)}\right\}_{i=1}^{\infty}$ which satisfies

$$
\begin{aligned}
& p_{n(i)-2 \tau}, p_{n(i)-2 \tau+1}, \ldots, p_{n(i)-\tau-1} \geq 0 \\
& 0 \leq p_{n(i)-\tau}, p_{n(i)-\tau+1}, \ldots, p_{n(i)-1}<1
\end{aligned}
$$

and

$$
p_{n(i)} \geq \Pi_{k=1}^{\tau} \Pi_{j=1}^{k}\left(1-p_{n(i)-j}\right)^{1 / \tau}
$$

for $i \geq 1$, then (1) cannot have any eventually positive solutions.

Proof. Assume to the contrary that $\left\{x_{n}\right\}$ is an eventually positive solution of (1). Then there exists a positive integer $I$ such that $x_{k}>0$ for $k \geq n(I)-3 \tau$. By means of Lemma 1, we see that

$$
x_{n(I)} \leq\left(1-p_{n(I)-1}\right) x_{n(I)-1}, \ldots, x_{n(I)-\tau+1} \leq\left(1-p_{n(I)-\tau}\right) x_{n(I)-\tau} .
$$

Thus

$$
\begin{aligned}
x_{n(I)} & \leq \Pi_{j=1}^{\tau}\left(1-p_{n(I)-j}\right) x_{n(I)-\tau}, \\
x_{n(I)} & \leq x_{n(I)-1} \leq \Pi_{j=1}^{\tau-1}\left(1-p_{n(I)-j}\right) x_{n(I)-\tau} \\
& \vdots \\
x_{n(I)} & \leq x_{n(I)-\tau+1} \leq\left(1-p_{n}(I)-\tau\right) x_{n(I)-\tau} .
\end{aligned}
$$

It follows that

$$
x_{n(I)}^{\tau} \leq x_{n(I)} x_{n(I)-1} \cdots x_{n(I)-\tau} \leq \Pi_{k=1}^{\tau} \Pi_{j=1}^{k}\left(1-p_{n(I)-j}\right) x_{n(I)-\tau}^{\tau},
$$

which implies that

$$
x_{n(I)} \leq \Pi_{k=1}^{\tau} \Pi_{j=1}^{k}\left(1-p_{n(I)-j}\right)^{1 / \tau} x_{n(I)-\tau} .
$$

In view of (1),

$$
\begin{aligned}
& 0<x_{n(I)+1} \leq x_{n(I)}-p_{n(I)} x_{n(I)-\tau} \\
\leq & {\left[\Pi_{k=1}^{\tau} \Pi_{j=1}^{k}\left(1-p_{n(I)-j}\right)^{1 / \tau}-p_{n(I)}\right] x_{n(I)-\tau} \leq 0, }
\end{aligned}
$$

which is a contradiction.

We can weaken the assumption that $p_{n(i)-\tau}, p_{n(i)-\tau+1}, \ldots, p_{n(i)-1}<1$ in the above Theorem, provided that the other assumptions are strengthened. Indeed, in view of Theorem 1, we see that the following result of Yu, et al. [2, Theorem 4] holds. 
Corollary 1. If $\left\{p_{n}\right\}$ has a subsequence $\left\{p_{n(i)}\right\}_{i=1}^{\infty}$ which satisfies

$$
\begin{aligned}
& p_{n(i)-2 \tau}, p_{n(i)-2 \tau+1}, \ldots, p_{n(i)-1} \geq 0, \\
& p_{n(i)-\tau}+p_{n(i)-\tau+1}+\cdots+p_{n(i)-1}<1
\end{aligned}
$$

and

$$
p_{n(i)} \geq 1-p_{n(i)-\tau}-p_{n(i)-\tau+1}-\cdots-p_{n(i)-1}
$$

for $i \geq 1$, then $(1)$ cannot have any eventually positive solutions.

For reasons to be provided later, we will give an alternate proof of this result as follows. Assume to the contrary that $\left\{x_{n}\right\}$ is an eventually positive solution of (1). Then there exists a positive integer $I$ such that $x_{k}>0$ for $k \geq n(I)-2 \tau$. If we now take $m=n(I)-\tau$ and $j=\tau-1$ in Lemma 1 , then we see that

$$
x_{n(I)} \leq\left(1-p_{n(I)-\tau}-p_{n(I)-\tau+1}-\cdots-p_{n(I)-1}\right) x_{n(I)-\tau} .
$$

In view of (1), we see further that

$$
\begin{aligned}
0 \leq & x_{n(I)+1} \leq x_{n(I)}-\frac{p_{n(I)} x_{n(I)}}{1-p_{n(I)-\tau}-p_{n(I)-\tau+1}-\cdots-p_{n(I)-1}} \\
& =x_{n(I)}\left\{1-\frac{p_{n(I)}}{1-p_{n(I)-\tau}-\cdots-p_{n(I)-1}}\right\} \leq 0
\end{aligned}
$$

which is a contradiction.

The same principles in the above proofs can be used to obtain several variants of the above Theorems. As an example, let us assume that $\left\{p_{n}\right\}$ has a subsequence $\left\{p_{n(i)}\right\}_{i=1}^{\infty}$
which satisfies

$$
p_{n(i)-3 \tau}, p_{n(i)-3 \tau+1}, \ldots, p_{n(i)-1} \geq 0
$$

If $\left\{x_{n}\right\}$ is an eventually positive solution of (1), then there exists a positive integer $I$ such that $x_{k}>0$ for $k \geq n(I)-4 \tau$. If we assume that

$$
\begin{array}{r}
\sum_{j=1}^{\tau} p_{n(i)-j-1}, \sum_{j=1}^{\tau} p_{n(i)-j-2}, \ldots, \sum_{j=1}^{\tau} p_{n(i)-j-\tau}<1, \\
\frac{p_{n(i)-1}}{1-\sum_{j=1}^{\tau} p_{n(i)-j-1}}, \frac{p_{n(i)-2}}{1-\sum_{j=1}^{\tau} p_{n(i)-j-2}}, \ldots, \frac{p_{n(i)-\tau}}{1-\sum_{j=1}^{\tau} p_{n(i)-j-\tau}}<1,
\end{array}
$$

as well as

$$
p_{n(i)} \geq \Pi_{k=1}^{\tau}\left[1-\frac{p_{n(i)-k}}{1-p_{n(i)-1-k}-p_{n(i)-2-k}-\cdots-p_{n(i)-\tau-k}}\right]
$$
for $i \geq 1$, then by means of the same reasoning used in the alternate proof of Corollary
1 , we see that

$$
\begin{aligned}
x_{n(I)} & \leq x_{n(I)-1}\left(1-\frac{p_{n(I)-1}}{1-p_{n(I)-2}-p_{n(I)-3}-\cdots-p_{n(I)-\tau}}\right), \ldots, \\
x_{n(I)-\tau+1} & \leq x_{n(I)-\tau}\left(1-\frac{p_{n(I)-\tau}}{1-p_{n(I)-1-\tau}-p_{n(I)-2-\tau}-\cdots-p_{n(I)-2 \tau}}\right) .
\end{aligned}
$$


Multiplying these inequalities together, we obtain

$$
x_{n(I)} \leq x_{n(I)-\tau} \Pi_{k=1}^{\tau}\left[1-\frac{p_{n(I)-k}}{1-p_{n(I)-1-k}-p_{n(I)-2-k}-\cdots-p_{n(I)-\tau-k}}\right] .
$$

In view of (1), we see further that

$$
\begin{aligned}
0 & <x_{n(I)+1} \leq x_{n(I)}-p_{n(I)} x_{n(I)-\tau} \\
& \leq x_{n(I)-\tau}\left\{\Pi_{k=1}^{\tau}\left[1-\frac{p_{n(I)-k}}{1-p_{n(I)-1-k}-\cdots-p_{n(I)-\tau-k}}\right]-p_{n(I)}\right\} \leq 0,
\end{aligned}
$$

which is a contradiction.

Other variants can be derived but no new principles are involved. Therefore, we now turn to an application of our results. Consider the following functional inequality

$$
y(t+\sigma)-y(t)+q(t) y(t-k \sigma) \leq 0,
$$

where $q \in C[0, \infty)$, and $\sigma, \delta>0$. Such an inequality has been studied by several authors, see e. g. [4]. By means of our Theorems, we will be able to find several sufficient conditions so that no eventually positive functions $y=y(t)$ can satisfy such an inequality for all large $t$. As an example, suppose $z=z(t)$ is such a continuous function. Then letting $z_{n}=z(n \sigma+T)$ and $q_{n}=q(n \sigma+T)$ for $n=1,2, \ldots$, we see that

$$
z_{n+1}-z_{n}+q_{n} z_{n-k} \leq 0
$$

for all large $n$. In view of Theorem 1 , if $q=q(t)$ has a subsequence $\left\{q_{n(i)}\right\}_{i=1}^{\infty}=$ $\left\{q\left(n_{i} \sigma+T\right\}_{i=1}^{\infty}\right.$ such that

$$
q_{n(i)-k}, q_{n(i)-k+1}, \ldots, q_{n(i)+j} \geq 0
$$

and

$$
q_{n(i)}+q_{n(i)+1}+\cdots+q_{n(i)+j} \geq 1
$$

for $i \geq 1$, then a contradiction will be reached.

\section{References}

[1] I. Györi and G. Ladas, "Oscillation theory of delay differential equations," Oxford Mathematical Monographs, Clarendon Press, Oxford, 1991.

[2] J. S. Yu, B. G. Zhang and X. Z. Qian, "Oscillations of delay difference equations with oscillating coefficients," J. Math. Anal. Appl., 177(1993), 432-444.

[3] C. J. Tian, S. L. Xie and S. S. Cheng, "Measures for oscillatory sequences," Computers Math. Appl., to appear.

[4] G. Ladas, L. Pakula and Z. Wang, "Necessary and sufficient conditions for the oscillation of difference equations," Pan Amer. Math. J., 2(1)(1992), 17-26.

Department of Mathematics, Datong Advanced College, Datong, Shanxi 037008, P. R. China. Department of Mathematics, Tsing Hua University, Hsinchu, Taiwan 30043, R. O. C. 